Sessions d'étude - Société canadienne d'histoire de l'Église catholique

\title{
L’organisation hospitalière dans le nord-ouest québécois
}

Résumé de la communication préparée pour le Congrès, Cégep

de Rouyn - 18 septembre 1982

\section{Simone Gareau}

Volume 49, 1982

URI : https://id.erudit.org/iderudit/1007090ar

DOI : https://doi.org/10.7202/1007090ar

Aller au sommaire du numéro

Éditeur(s)

Les Éditions Historia Ecclesiæ Catholicæ Canadensis Inc.

ISSN

0318-6172 (imprimé)

1927-7067 (numérique)

Découvrir la revue

Citer cet article

Gareau, S. (1982). L'organisation hospitalière dans le nord-ouest québécois : résumé de la communication préparée pour le Congrès, Cégep de Rouyn - 18 septembre 1982. Sessions d'étude - Société canadienne d'histoire de l'Église catholique, 49, 35-58. https://doi.org/10.7202/1007090ar

Tous droits réservés @ Les Éditions Historia Ecclesiæ Catholicæ Canadensis Inc., 1982
Ce document est protégé par la loi sur le droit d'auteur. L'utilisation des services d'Érudit (y compris la reproduction) est assujettie à sa politique d'utilisation que vous pouvez consulter en ligne.

https://apropos.erudit.org/fr/usagers/politique-dutilisation/ 


\section{L'organisation hospitalière dans le nord-ouest québécois}

Résumé de la communication préparée pour le Congrès

Cégep de Rouyn - 18 septembre 1982

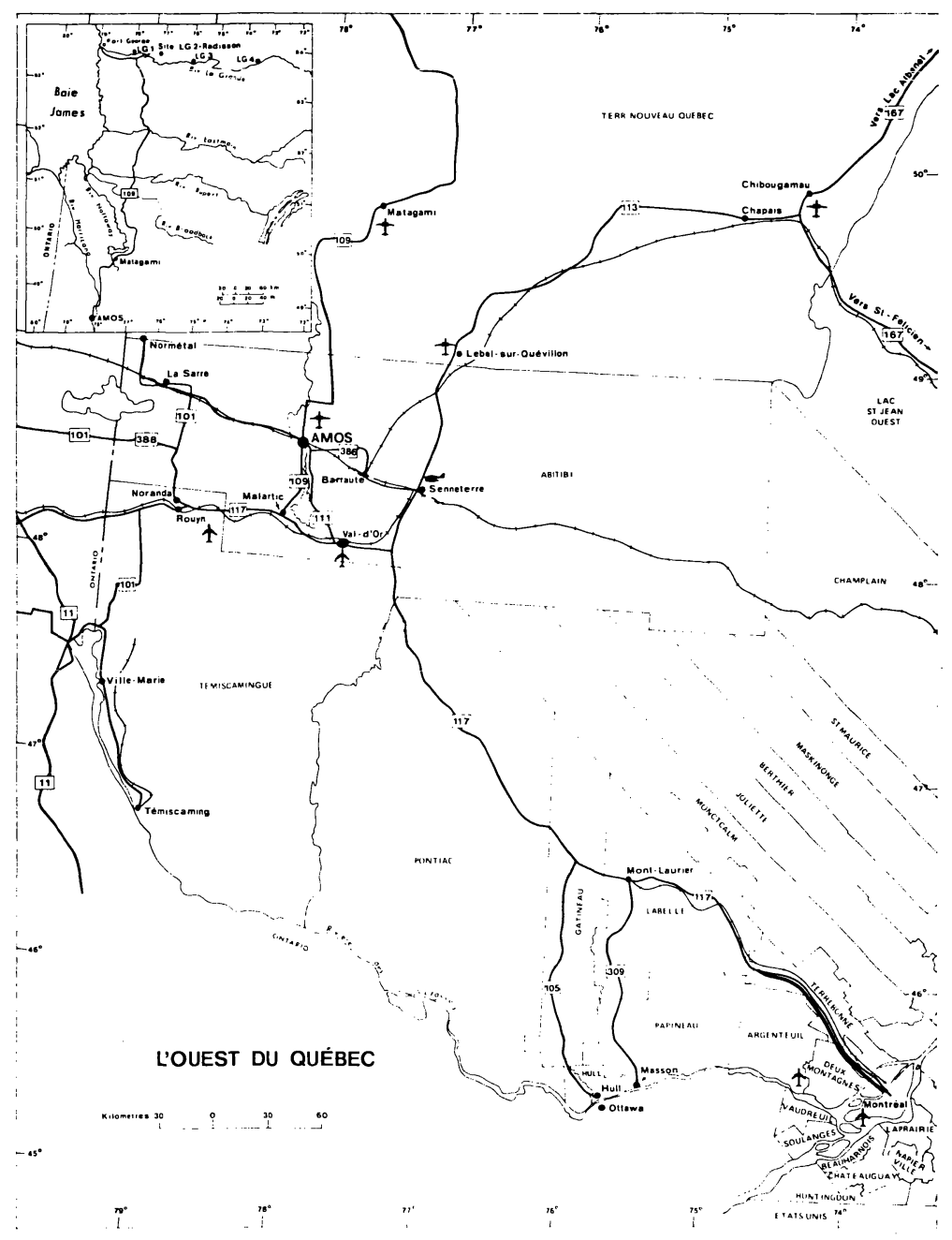


J'ai à exposer devant vous le travail accompli auprès des malades dans la région de l'Abitibi-Témiscamingue connue aujourd'hui sous le nom de Nord-ouest québécois. Je brosserai un tableau de l'installation et de l'évolution de l'oeuvre hospitalière à Ville-Marie. Rouyn-Noranda, Amos, Val d'Or, Macamic, La Sarre, Malartic et Fort George. à la Baie James.

\section{LA MISSION STE-CLAUDE (1866)}

L'année 1866 marque une date importante dans l'histoire de la Congrégation des Soeurs Grises d'Ottawa : celle de la première fondation missionnaire en terre canadienne, la mission Ste-Claude du Témiscamingue. Elle a lieu chez les Algonquins, à l'entrée du Lac Témiscamingue, véritable mer intérieure formée par l'élargissement de la rivière Outaouais à 300 milles au nord de la capitale du Canada. Même si cette fondation commence en terre ontarienne, elle retient notre attention, car elle est à l'origine du développement de son rejeton en terre québécoise : Ville-Marie. La mission Ste-Claude ou du Vieux Fort Témiscamingue, sur la rive ontarienne du Lac Témiscamingue, voit le jour en 1862, grâce à l'autorisation donnée par $\mathbf{M}^{\mathrm{gr}}$ Ignace Bourget de Montréal, de fonder une mission permanente au Fort Témiscamingue. Cet endroit où les rives du lac viennent se rejoindre pour ne laisser libre qu'une étroite passe, constitue un point stratégique pour l'évangélisation de l'immense territoire confié à l'apostolat des Oblats de Marie-Immaculée.

En 1866, le Père Pian, de la mission Ste-Claude, présente une supplique secondée par $\mathbf{M}^{\mathrm{gr}}$ Bruno Guigues, évêque d'Ottawa, au Conseil général des Soeurs Grises de la Croix, afin d'obtenir des religieuses pour partager le travail apostolique des missionnaires. Les Soeurs Raizenne et St-Vincent acceptent de relever le défi. Elles quittent les rives de l'Outaouais à Aylmer sur un bateau à vapeur: le Pontiac, le 2 octobre 1866.

Il leur aura fallu quinze jours de bateau, de canots, de portage, de marches pénibles dans la forêt, pour franchir les trois cent quatorze milles qui les séparaient de la Capitale. Missionnaires, Blancs, Indiens, accueillent avec joie les nouvelles recrues arrivées le 18 octobre 1866. Les débuts sont marqués d'héroïsme quotidien : maison de billots équarris, locaux exigus, pauvreté extrême, salaire inexistant pendant dix ans. Les soeurs s'improvisent cultivateurs afin de pourvoir à la nourriture du personnel de la Mission. Le 7 décembre 1866, elles fondent le premier hôpital formé d'une seule pièce au bout de la cuisine. La première 


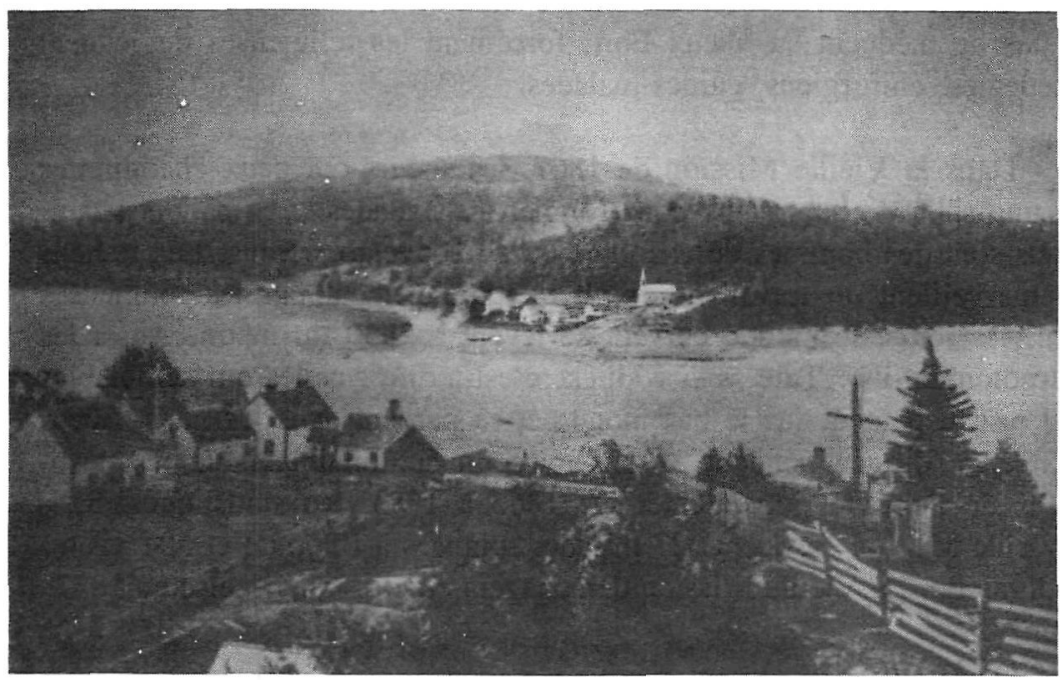

(ca 1867)

Au premier plan, le poste de traite de la Compagnie de La Baie d'Hudson avec la tombe du Père Laverlochère.

La Croix de droite fut plantée par $M^{g r}$ Bruno Guigues, le 15 août 1864, lors de la première visite épiscopale dans le Témiscamingue.

Sur l'autre rive : la mission catholique Ste-Claude.

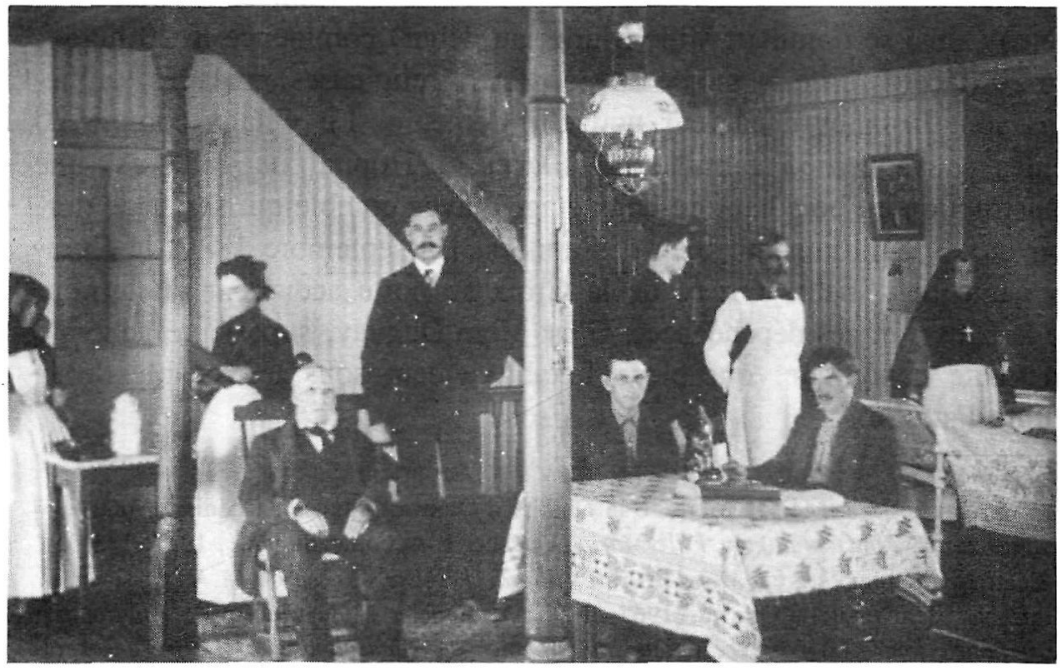

La saille du vieil hôpital en 1897 : à gauche : S. Ste-Rosalie, à droite : S. St-Basile. 
patiente, c'est une vieille indienne du nom de Catherine. Comme il n'y a pas de médecin au Vieux Fort, forcément les religieuses deviennent médecins, chirurgiens, gardes-malades.

Dans la Vieille Mission de 1867, les soeurs ont deux chambrettes et une salle de quatre lits pour soigner les malades. Les patients habituels sont des hommes de chantiers ou de nouveaux colons. Les Indiens s'émerveillent du travail accompli quotidiennement par les religieuses qui, les jours de grande corvée, se lèvent à $2 \mathrm{~h} 30$ du matin. Aussi les surnomment-ils : Taie sana widjick, c'est-à-dire : celles qui semblables à l'oiseau planent sur le bas monde et se perdent dans les nues. Soeur Raizenne dirigea pendant 22 ans avec un entrain inlassable les destinées de l'oeuvre polyvalente. De 1866 à 1883, les statistiques de la «Vieille Mission» révèlent 327 malades soignées à l'hôpital et 504 visites à domicile. La Vieille Mission déménagera en terre québécoise en 1887.

\section{L'HÔPITAL SAINTE-FAMILLE DE VILLE-MARIE (1887) ${ }^{1}$}

Grâce à la ténacité du Frère Moffet, défricheur du premier lopin de terre sur la terre québécoise en 1879 , une aube nouvelle se lève pour l'oeuvre commencée et poursuivie par les Soeurs Grises de la Croix sur la rive ontarienne. Le 19 mai 1887, la construction de l'église, du presbytère, de l'Hôpital est commencée du côté québecois, sous la direction du Frère Lapointe. Le déménagement de la mission à la Baie des Pères (ainsi se nommait Ville-Marie, au début) commence le 20 juillet. Le 20 décembre de la même année, Pères, religieuses, malades, orphelins sont installés dans leur nouvelle demeure. Le village compte 20 tentes sauvages, 35 familles de Blancs. L'Hôpital de 1887 est une construction en bois haut de deux étages, avec comble qui servit de dortoir aux Soeurs pendant bien des années. En septembre 1889, la Communauté des Soeurs Grises de la Croix se porte acquéreur du terrain, de la bâtisse et des dépendances. C'est Mère Duguay, supérieure générale en visite canonique, qui place l'établissement sous le vocable de la Sainte-Famille.

${ }^{1}$ Archives de la Maison-Mère de la Charité d'Ottawa; Augustin Chénier, Notes historiques sur le Témiscamingue, Ville-Marie (Québec, L'Action catholique, 1937, 133 p.); Sr Béatrice Cloutier, s.g.c., Mère Bruyère, fille de l'Église (Thèse de M.A. en sciences religieuses, Université d'Ottawa, 1967, 110 p.); Eugène Nadeau, o.m.i., Un homme sortit pour semer. La carrière épique du pionnier du Témiscamingue. Le frère Joseph Moffet, o.m.i. (1852-1932) (Montréal, Fides, 1951, 195 p.); Sr Paul-Émile, s.g.c., Mère Elisabeth Bruyère et son oeuvre. Les Soeurs Grises de la Croix, t. I, 1845-1876 (Ottawa, Maison-Mère, 1945, 409 p.). 
Dès le 10 mai 1886 , un Comité de 24 paroissiens change le nom de Baie-des-Pères en celui de Ville-Marie. En 1897, Ville-Marie est érigée en municipalité. L'Hôpital de Ville-Marie est reconnu hôpital régional depuis 1887 . Les patients y viennent de tous les villages du Témiscamingue. Pendant l'année 1923, 395 malades sont hospitalisés.

En 1926, on ajoute une annexe dont le coût est supporté par la Communauté des Soeurs Grises. Le Gouvernement paie l'intérêt des obligations. Les traitements, les remèdes sont fournis gratuitement ainsi que la lingerie, les assurances, les appareils. Les religieuses ne se réservent aucun salaire. L'établissement agrandi compte 40 lits répartis en dix chambres privées et deux salles publiques, deux salles d'opération, un laboratoire, un département de radiologie et de physiothérapie. Le personnel comprend trois médecins, huit gardes-malades dont quatre religieuses.

Au temps de la crise économique des années '30, la situation devient précaire. L'argent manque: $\$ 20,000$ de compte non-payés, $\$ 9,000$ de déficit de caisse. Les religieuses s'imposent un régime de légumes et de pain sec. Elle conservent les petites provisions de viande, d'oeufs, de beurre pour les patients. Pendant des mois, infirmières de jour ou de nuit s'imposent un jeûne rigoureux. ${ }^{2}$ L'hôpital réussit à sortir de l'impasse, grâce à des subventions spéciales.

Les statistiques de 1940 indiquent: 613 malades hospitalisés, 162 interventions chirurgicales majeures, 197 mineures, 140 radiographies, 300 laboratoires et 265 traitements en physiothérapie. En 1951, le vieil hôpital de 1887 tombe sous le pic des démolisseurs. On édifie l'annexe actuelle qui fait l'orgueil de la région témiscamienne.

Mais l'histoire présente parfois des virages subits et bouleversants. Arrive l'époque de la révolution tranquille des années 60-70 qui transforme d'abord le système d'éducation puis l'organisation hospitalière à travers la Province. Toutes les institutions relevant du Ministère des Affaires sociales doivent refondre la structure de leur Corporation selon les exigences de la loi 48, entrant en vigueur le ler janvier 1973. Les communautés religieuses perdent l'une après l'autre la gestion de leurs hôpitaux, de leur foyers de vieillards, de leurs orphelinats; l'État en devient le propriétaire moyennant une certaine indemnité. L'Hôpital

${ }^{2}$ Extrait d'Augustin Chénier, Notes historiques sur le Témiscamingue, Ville-Marie, 1937, p. 41 et suiv. 
Sainte-Famille ne souffre pas d'exception. En 1974, l'État recueille les fruits de 108 années de dévouement et de sacrifices.

Le Centre de santé Sainte-Famille de Ville-Marie (d'après la nouvelle appellation) a admis 1619 patients en 1981. Sept médecins et un chirurgien, 25 infirmières autorisées et 50 gardes-malades auxiliaires y oeuvrent avec l'aide de 29 employés de soutien. Il n'y reste que deux religieuses à temps plein.

\section{L'HÔPITAL DES SAINTS ANGES (YOUVILLE) DE ROUYN-NORANDA $(1926)^{3}$}

Après Ville-Marie, c'est Rouyn qui prend un essor vertigineux dans l'espace de quelques mois. Grâce à la perspicacité de $\mathbf{M}^{\mathrm{gr}}$ Louis Rhéaume, évêque d'Haileybury et du curé Albert Pelletier arrivé à Rouyn le 7 juin 1925, le Conseil général des Soeurs Grises de la Croix accepte d'envoyer deux religieuses pour l'école et deux pour le soin des malades le 27 septembre 1925. Se rendre à Rouyn en 1925, c'était une aventure aussi ardue que de faire le trajet d'Ottawa à la mission Ste-Claude en 1866. Les fondatrices, Soeur Ste-Marcelline, supérieure, pharmacienne et responsable du dispensaire et Soeur M.-Elmire, infirmière, Soeur Ste-Judith et Charles-Ovide institutrices, partent de la gare Union d'Ottawa dans la soirée du 20 octobre où le Canadien Pacifique les conduit jusqu'à Mattawa. À cet endroit, leurs consoeurs de l'Hôpital SaintJoseph, fondé en 1870, les accueillent et leur offrent quelques articles pour le futur Hôpital de Rouyn.

Le 21 , le train les amène jusqu'à Ville-Marie où $\mathrm{M}^{\mathrm{gr}}$ Rhéaume se fait un devoir de venir les saluer et les encourager. Le 22, les fondatrices se rendent à Angliers en automobile. Les routes sont tellement cahoteuses qu'il leur faut cinq heures pour franchir la distance de 31 milles. Harassées, les voyageuses acceptent l'hospitalité de M. J. Boisvert, responsable des gardes-feux de la région. Dès $7 \mathrm{~h} 00 \mathrm{du}$ matin le 23 octobre on entreprend le parcours de 110 milles en bateau à

\footnotetext{
${ }^{3}$ Sources consultées : Archives de la Communauté des Soeurs de la Charité d'Ottawa; Archives de la Société historique du Nord-Ouest québécois; Procès-verbaux de l'Hôpital de Noranda; The Northern Daily News, 1950; Journal La Frontière, 1951 et 1965; Charles Ladouceur, Album-souvenir de Rouyn-Noranda, 1924-1939 (Ottawa, Commercial Press, 1939, 114 p.); Albert Leury, Histoire de Rouyn-Noranda, t. III : Écoles et Institutions (1939); $\mathrm{M}^{\mathrm{gr}}$ Albert Pelletier, J'ai vu naître et grandir ces jumelles (RouynNoranda), (Val d'Or, 1967, 195 p.).
} 
gazoline. Arrêt aux Rapides l'Esturgeon pour un portage de trois-quarts de mille dans la glaise détrempée. Å $13 \mathrm{~h} 00$, départ de l'autre embarcation qui coule sur les eaux de la rivière Ottawa, Kinovéjis, les lacs Caron, Vallet, Routhier, Rouyn. La glace est prise sur le lac Rouyn. La neige tombe drue et rend la visibilité presque nulle. Vers $17 \mathrm{~h} 00$, le bateau échoue sur le sable à un demi mille plus bas que l'entrée du chenal qui relie les lacs Routhier et Rouyn. Le moral est à la baisse. C'est alors que l'intrépide navigateur, le curé Pelletier, vient à la rescousse de l'équipée: il prend le gouvernail et traverse le lac Rouyn jusqu'au camp de $\mathbf{M}$. Augustin Chénier. Un portage d'un mille et demi amène les passagères au bord du lac Osisko. Une rapide embarcation les conduit au camp des gardes-feux : un chantier de 20 pieds par 16 qui sera leur habitation jusqu'à la mi-novembre. Il est $23 \mathrm{~h} 30$. On serait exténué à moins.

La visite des lieux, le 24 octobre, permet aux religieuses de faire connaissance avec leur entourage. Même si l'école-chapelle à deux étages située sur les bords du lac Osisko est encore en construction, l'espérance de jouir bientôt d'une salle de classe et d'une salle à tout usage y compris le dispensaire, les remplit de joie. Dès que les caisses de remèdes arrivent, le dispensaire ouvre ses portes. Nous sommes au 26 novembre. L'histoire a retenu les noms des Docteurs Linklater, Rudd, Bouillé, Breault, Cartier et du dentiste J.V. Smith qui ont bravé l'éloignement et la vie facile des grands centres pour se dévouer auprès des mineurs et des bûcherons du Nord-Ouest québécois.

Le 17 janvier 1926, le curé Pelletier annonce la construction d'un hôpital sur le terrain voisin de l'école. De son côté, $\mathrm{M}^{\mathrm{gr}}$ Rhéaume obtient du gouvernement provincial la promesse d'un octroi triennal de $\$ 5,000$. Les travaux vont bon train, si bien que $\mathbf{M}^{\mathrm{gr}}$ l'évêque bénit l'hôpital à peu près terminé le 26 septembre 1926 et le place sous le vocable des Saints Anges.

La population admire cette bâtisse de 74 x 34 pieds d'une capacité de 30 lits environ. Sont à la disposition des malades, deux chambres privées, deux chambres semi-privées, deux salles publiques, un département de maternité, une pouponnière avec quatre berceaux et une salle d'opération. Le 25 novembre, les Soeurs quittent l'unique pièce de l'école-chapelle pour habiter l'Hôpital régional des Saints Anges, propriété de la Corporation épiscopale du diocèse d'Haileybury. La mine de Noranda dote l'institution d'un appareil de radiographie. 


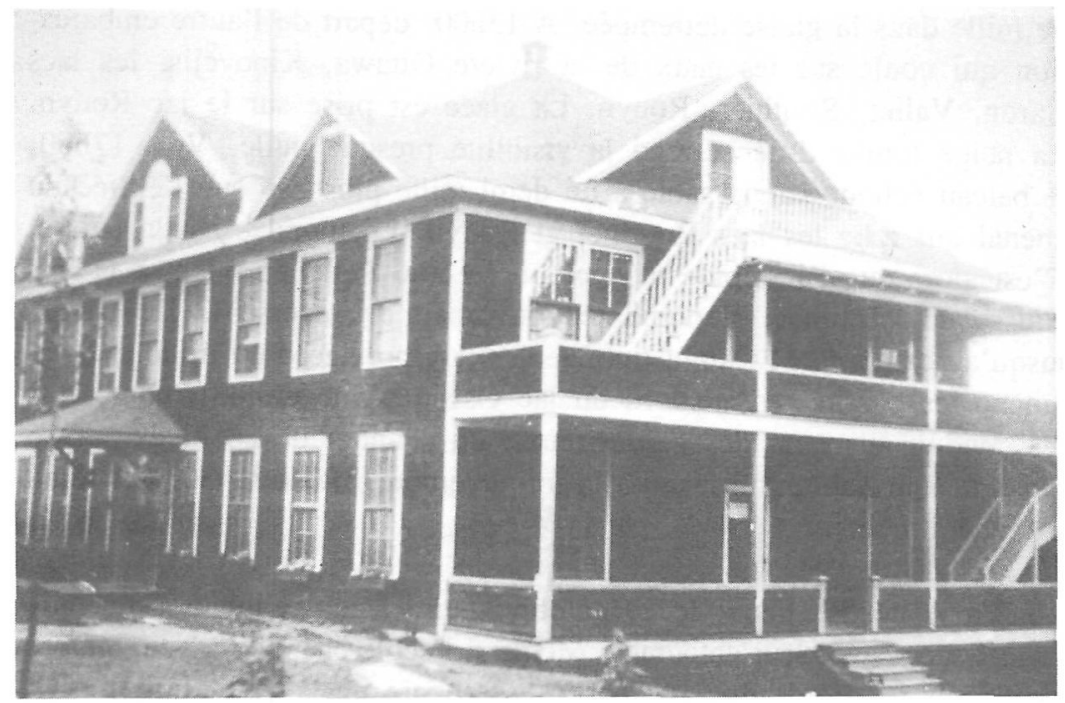

L'hôpital des Saints-Anges de 1926 à 1929

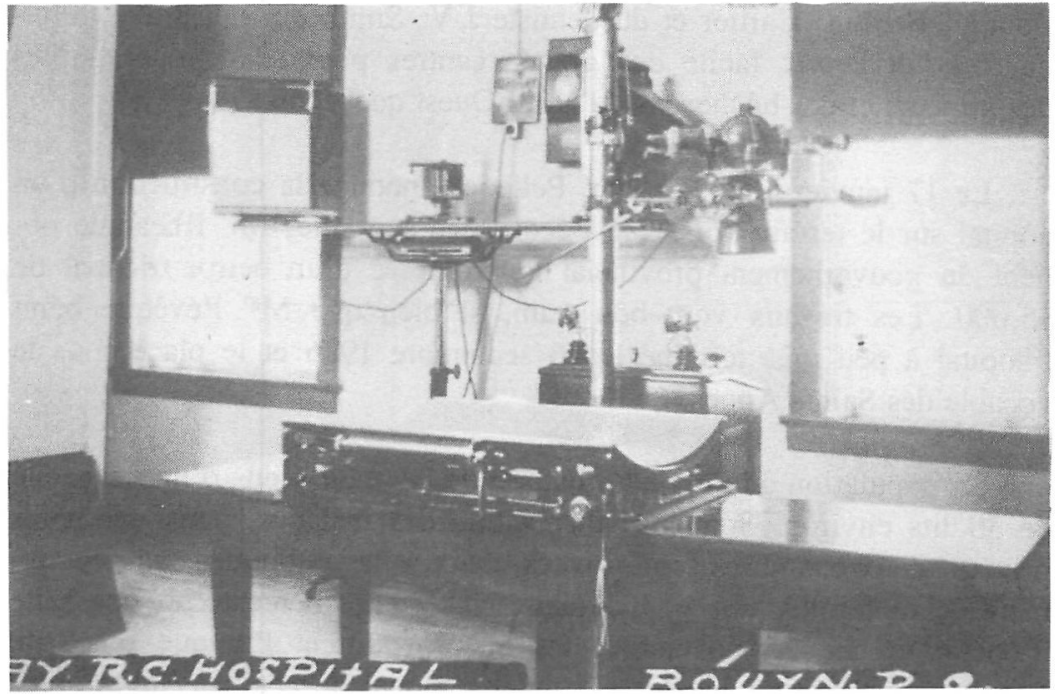

Le département de radiographie 1926-29 
Rouyn se développe de façon prestigieuse, puisque ce centre minier est érigé en municipalité en 1926 et en paroisse sous le vocable StMichel, le 25 avril 1927. La ville soeur Noranda (contraction de Nord Canada) en fait autant. James Murdoch, président de la Noranda Mines, suggère aux responsables de l'hôpital des Saints Anges d'ériger un autre hôpital plus près de la mine. "C'est prématuré» dit-on. $\mathrm{M}^{\mathrm{gr}}$ Rhéaume fait agrandir l'hôpital à peine aménagé afin d'y accueillir 45 malades. Cette deuxième étape est de courte durée. Devant l'évidente insuffisance de lits et la possibilité de transformer cet immeuble en Pensionnat pour jeunes filles, cette fois c'est la Congrégation des Soeurs Grises de la Croix qui consent en mars 1928 à édifier un hôpital plus vaste sur un terrain cédé par la Corporation minière de Noranda et situé aux limites des villes jumelles. Le prospecteur de la mine, Edmond Horne, offre un montant de $\$ 20,000$ pour l'aménagement d'une salle d'opération. La bâtisse de quatre étages plus un rez-de-chaussée aura 108 pieds par 54, pour une capacité de 100 lits.

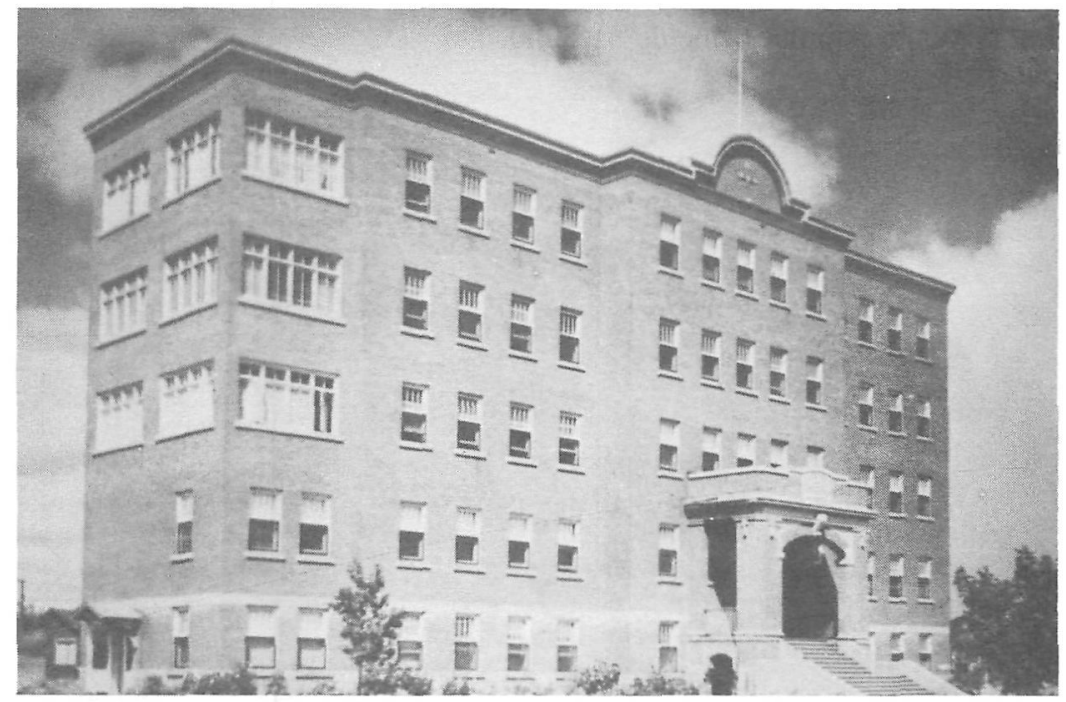

Hôpital Youville (1929)

Le 20 août 1930 s'opère le transfert des malades de l'hôpital des Saints Anges au nouvel hôpital Youville. Voici les statistiques pour l'année 1931 : 1092 patients admis, 10,285 jours d'hospitalisation, 597 cas médicaux, 364 cas chirurgicaux, 71 naissances, 2050 RX miniers. En novembre 1931, la mine de Noranda a loué le département de radiologie à raison de $\$ 200$ par mois pour le dépistage de la silicose chez les mineurs. Elle fournit les services du Dr McArthur de l'hôpital Royal Victoria de Montréal. Le personnel comprend : 15 religieuses, 9 méde- 
cins, 5 gardes-malades licenciées et 15 gardes-malades auxiliaires. L'année suivante on ajoute la clinique pour les maladies des yeux, du nez et des oreilles.

En 1940, l'hôpital enregistre 3184 admissions, en 1947 : 4402 . On construit une résidence sur le terrain de l'hôpital pour loger les employés, afin de libérer des chambres pour les patients. Le personnel de 1944 comprend : 25 religieuses, 5 gardes-malades graduées, 16 gardesmalades auxiliaires, 35 employés féminins, 2 infirmiers et 6 employés masculins.

Devant l'urgence de la situation, la Congrégation des Soeurs Grises de la Croix ne peut rester indifférente et décide d'agrandir. Le 22 juin 1945, elle permet l'ajout de deux étages supplémentaires à l'hôpital existant. Le ministre de la Santé, Albini Paquette en visite à RouynNoranda, promet une aide financière substantielle pour cet agrandissement majeur. En 1947, la Noranda Mines fait don de $\$ 60,000$ à 1'hôpital et la Waite Amulet Mine de $\$ 15,000 .^{4}$

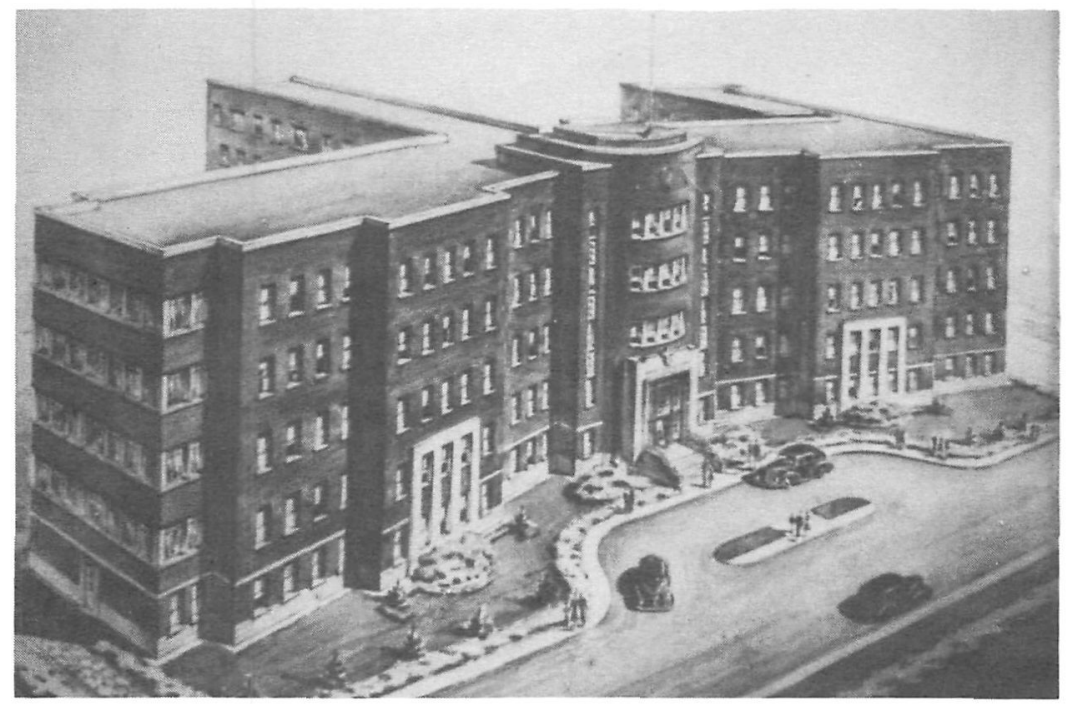

Hôpital Noranda (1946)

${ }^{4}$ Lors du 25e anniversaire de fondation de l'Hôpital, la Noranda Mines offre un cadeau de $\$ 40,000$ et la Waite Amulet de $\$ 10,000$. 
Le contrat avec la Maison Collet et Frères de Montréal en 1946 compoite la restauration de la façade qui mesure 280 pieds; la construction de deux ailes de 108 pieds de long, un motif central de 50 pieds plus un solarium de 12 pieds. La capacité sera portée à 225 patients avec une possibilité de 280 . En 1948 , les travaux sont terminés. La masse architecturale fait l'orgueil des citadins des villes-soeurs et de la population de 60,000 habitants distribuée dans les comtés Témiscamingue-Abitibi.

La difficulté d'obtenir des compétences de l'extérieur, vu l'éloignement des grands centres, décide les autorités de l'hôpital avec le concours des médecins d'ouvrir une école de gardes-malades en 1928. Le nombre d'aspirantes augmente d'année en année, si bien que l'École d'Infirmières reçoit un diplôme d'affiliation de l'Université Laval de Québec. Malheureusement à cause du manque de préparation académique des aspirantes du milieu, l'École doit former des gardes-malades pratiques durant quelques années. En 1950, la direction de l'École juge les aspirantes suffisamment préparées pour continuer le cours de gardesmalades licenciées. En 1953, douze nouvelles infirmières reçoivent leur diplôme de l'Université Laval. Ces recrues rendent un service de premier ordre à l'hôpital qui en 1955 a admis 7444 patients pour 71,310 jours d'hospitalisation. Les 24 religieuses, les 28 gardes-malades enregistrées, les 31 gardes-malades étudiantes, les 17 gardes-malades auxiliaires sans compter les 42 aides et les 88 employés suffisent à peine à répondre aux besoins de la clientèle.

Le succès toujours grandissant de l'École d'infirmières, particulièrement sous la direction de Soeur Gabrielle Laramée qui s'y dévoua pendant 20 ans, nécessite un espace mieux adapté au bien-être des étudiants. En 1965, une Résidence des Infirmières au coût de $\$ 2,000,000$ s'élève sur le terrain de l'hôpital et comporte une piscine intérieure, la première dans les institutions de la région.

Mais avec la restructuration scolaire des années ' 60 , l'avènement des Collèges d'Enseignement général et professionnel a absorbé toutes les formes d'enseignement de niveau pré-universitaire. L'École des infirmières de Rouyn-Noranda n'a pas échappé à cette réforme. Le Pavillon Laramée sert maintenant de bureaux à la Sûreté du Québec tandis que la ville de Noranda loue la piscine et le gymnase.

Et l'hôpital de 1948 a-t-il tenu le coup ? Devant l'évolution rapide de la médecine et les progrès de la technologie, l'hôpital Youville ne répond plus aux besoins de l'heure. Les autorités gouvernementales, 
le corps médical et hospitalier le trouve non-fonctionnel. Le Ministère des Affaires sociales approuve les plans du Centre hospitalier RouynNoranda (Pavillon Lemay-Juteau), édifice de six étages situé au bord du lac Osisko. Terminé en 1973, ce Centre peut recevoir 240 patients et 38 poupons. Le personnel de 1982 comprend 35 médecins dont 14 spécialistes, sans compter les 12 autres qui viennent de Montréal chaque mois pour prêter main forte à leurs confrères.

Que devient l'hôpital Youville ? Il abrite le Département de santé communautaire, le centre régional des services de santé et sociaux (CRSSS), les cours pré-nataux, les services de soins à domicile, le planning des naissances, un département de psychiatrie et d'ergothérapie, le service social pour les patients hospitalisés; 75 lits pour personnes handicapées ou pour malades chroniques en projet pour 1983.

La Communauté des Soeurs Grises de la Croix (Soeurs de la Charité d'Ottawa) a conservé la gestion de l'hôpital jusqu'en 1973. Par la loi 48 sanctionnée en décembre 1971, toutes les institutions relevant du Ministère des Affaires sociales ont dû accepter de refondre la structure de leur Corporation. Le Ministère a versé le 1/6 de la valeur investie par la Congrégation religieuse propriétaire et devient le maître de céans. Actuellement une seule religieuse remplit le poste d'archiviste.

C'est la fin d'une aventure apostolique d'envergure pour les Soeurs de la Charité d'Ottawa, c'est le mystère de la destinée des oeuvres qui naissent péniblement et meurent en douce.

\section{L'HÔPITAL STE-THÉRÈSE (HÔTEL-DIEU) D'AMOS (1930) ${ }^{5}$}

Quand on parle de l'Abitibi, on ne peut oublier l'ère de colonisation des années de la fameuse crise économique des années '30. Les colons y ont trimé dur sur des terres souvent peu propices à la culture, ont fondé plusieurs villages dont la plupart subsistent encore. En 1978, dans la région d'Amos, il existait 67,149 hectares de terres défrichées occupées par 342 cultivateurs. Amos, connu comme le «berceau de l'Abitibi", est la première municipalité à être incorporée en 1912. Elle fut érigée en diocèse le 3 décembre 1938. $\mathrm{M}^{\mathrm{gr}} \mathrm{J}$. Aldée Desmarais en fut le premier évêque (1939-1968).

${ }^{5}$ Sources consultées : Archives médicales de l'Hôtel-Dieu d'Amos; Dossier économique d'Amos; Journal Le Reflet, février 1981; Yvette Paquin, s.g.m., Manuscrit pour le 50e anniversaire de l'Hôtel-Dieu d'Amos, 1980. 
Venons-en à l'organisation hospitalière de ce coin de pays. L'abbé Viateur Dudemaine, curé d'Amos, ambitionne d'obtenir un hôpital qui desservirait la municipalité et ses environs. En 1918, il rencontre à maintes reprises les Soeurs Grises de Nicolet. ${ }^{6}$ Les lendemains de la guerre 1914-1918 ne sont pas très reluisants au point de vue économique. La communauté sollicitée est prête à accepter la demande du curé, mais hésite à endosser le fardeau financier d'une telle construction. Le pasteur n'abandonne pas son projet. Il revient à la charge et finit par obtenir l'assentiment du Conseil général des Soeurs Grises, après onze années d'attente. Le Conseil de ville fait don d'un terrain sur les bords de la rivière Harricana. Commencé en 1929, l'hôpital Ste-Thérèse ouvre ses portes le 14 mai 1930. De généreux donateurs aident à l'aménagement des chambres et des divers départements. Huit religieuses forment le premien contingent: les Soeurs Alphonsine Normand, supérieure, M.-Anne Théroux, Eveline Boudreault, Niama Guertin, Irénia Limoges, Robertine Goudreault, Duguay et St-Luc.

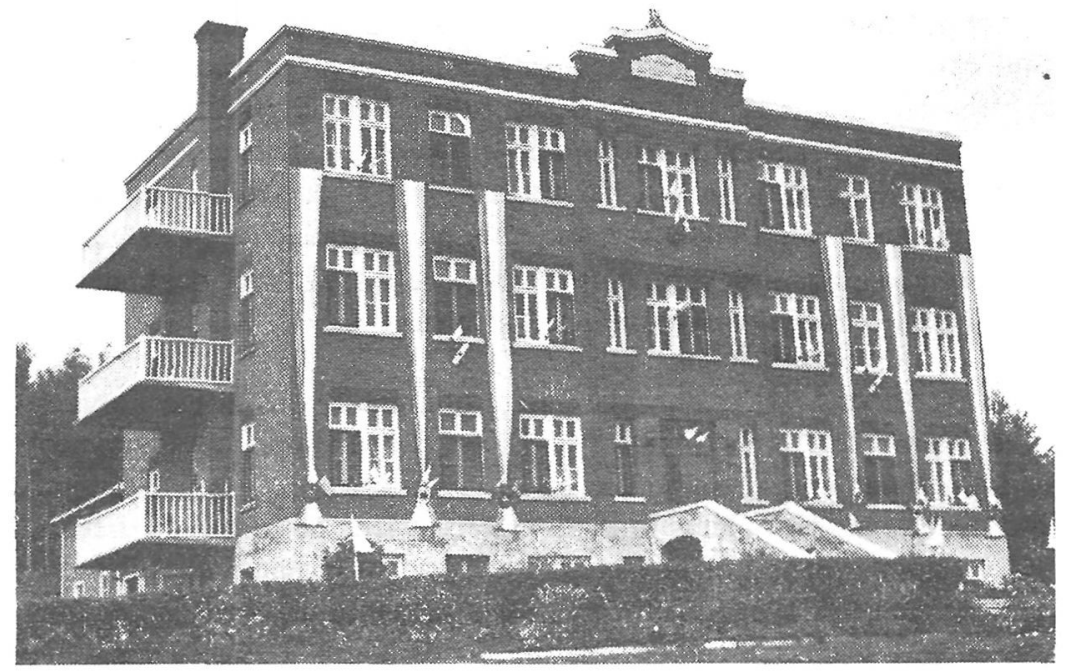

L'Hôpital Ste-Thérèse d'Amos, tel qu'il apparaissait une fois sa construction terminée, en 1930. Ce premier immeuble est maintenant occupé par le Centre d'Accueil Normand pour alcooliques et autres toxicomanes.

${ }^{6}$ Les Soeurs Grises de Nicolet sont un détachement de la communauté des Soeurs Grises de St-Hyacinthe en 1886; elles seront réunies aux Soeurs Grises de Montréal en 1940. Cf. Marguerite JEAN, Évolution des communautés religieuses de femmes au Canada de 1639 à nos jours, Montréal, Fides, 1977, p. 139-140 et 274-275. 


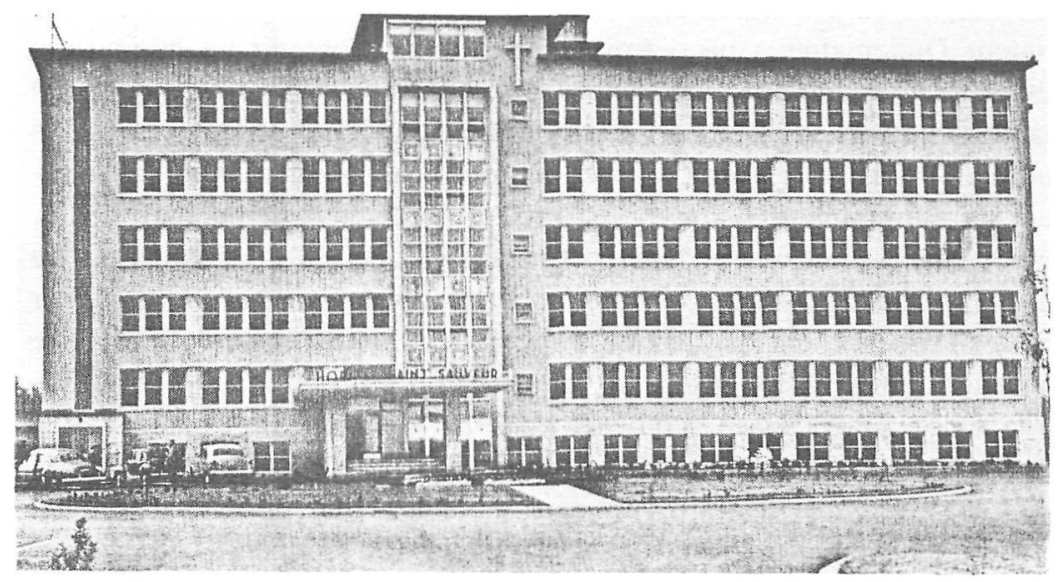

Hôpital St-Sauveur, Val d'Or.

On ne trouve pas toutes les commodités dans ce premier hôpital d'une capacité de 35 lits, mais les responsables tirent profit de tout pour suppléer au manque de confort. Grâce au prestige du chirurgien Joseph Dion, le jeune hôpital jouit bientôt d'une renommée enviable. Les statistiques de 1931 révèlent : 405 patients, 6,412 jours d'hospitalisation, neuf religieuses, cinq employés féminins, un infirmier et un aumônier. Un Comité de Dames patronnesses fondé en 1932 apporte une aide financière et sociale à l'institution peu fortunée.

C'est Soeur Marie-Anne Théroux qui durant 20 ans dirige l'organisation hospitalière de l'Hôpital : les salles d'opération, le département de physiothérapie, la pharmacie et le laboratoire. Les statistiques de 1940 indiquent 778 admissions. L'établissement devient trop petit... 1942 : l'hôpital Ste-Thérèse change son appellation pour celle d'HôtelDieu d'Amos. En 1949, un édifice plus spacieux d'une capacité de 250 lits s'élève à côté du premier. Quinze ans plus tard, 1'Hôtel-Dieu subit un agrandissement qui permet d'offrir 303 lits aux patients. Du 15 octobre 1959 au 21 mai 1964, il accueille 70,038 malades. L'ouverture d'une école de formation de gardes-malades auxiliaires en Nursing en 1950 permet à 331 jeunes filles d'obtenir un diplôme entre 1950 et 1971. Une école de technologie médicale en 1961 prépare 26 techniciennes en laboratoire.

En mars 1966, le Ministère de la Santé du Québec autorise la réfection intérieure et extérieure de la bâtisse, l'ajout de deux salles de chirurgie majeure, l'agrandissement de la cuisine et de la cafétéria, 
la construction de l'entrée principale. La population passe de 4,000 habitants en 1943 à 12,640 en 1981. Le personnel de l'hôpital comprend 300 personnes à temps complet, 88 à temps partiel régulier et 125 à temps partiel sur demande. En 1980, les statistiques déclarent : 4,132 admissions adultes et enfants et 570 bébés. Mais depuis 1972, les religieuses ne jouissent plus que du statut d'employées. L'étatisation des hôpitaux met fin à 50 ans d'administration marquée au coin de l'abnégation, du désintéressement et du souci des déshérités de la santé.

\section{L'HÔPITAL SAINT-SAUVEUR DE VAL D'OR (1950).}

Val d'Or, comme son nom l'indique, possède des mines du précieux métal et fait partie de l'Ábitibi. Cette ville fondée en 1934 et située à $106 \mathrm{~km}$ de Rouyn et à $67 \mathrm{~km}$ d'Amos s'est développée très rapidement. Dès 1946, un comité de citoyens réclame un hôpital à cause de l'expansion de la ville et des nombreux accidents de la mine. $\mathbf{M}^{\mathrm{gr}}$ Desmarais, évêque d'Amos, souhaite vivement un hôpital à Val d'Or.

Toutes ces pressions conjuguées aboutissent à l'événement du 28 mai 1950 alors que plusieurs autorités religieuses et civiles du diocèse d'Amos, du comté de l'Abitibi, de la ville de Val d'Or, de la communauté des Filles de la Sagesse, du Corps médical assistent à la bénédiction de la pierre angulaire du nouvel hôpital Saint-Sauveur, situé sur la 6e rue dans la partie ouest de la ville.

Mère Marie-Amédée de la Présentation fut la première supérieure de cet édifice imposant de six étages. En 1950, vingt-cinq religieuses oeuvrent à temps plein auprès des malades et voient à l'administration et à la bonne marche de l'établissement. À cette époque Val d'Or compte 8,000 habitants. Les statistiques de l'hôpital pour l'année de fondation démontrent: 2,176 patients hospitalisés et 165 patients externes. Dix ans plus tard, l'hôpital enregistre 5,280 patients hospitalisés, 1,559 patients externes, 34 religieuses, 7 infirmières graduées laïques, 11 gardesmalades auxiliaires, 14 médecins omnipraticiens et 7 spécialistes. Comme dans les autres centres hospitaliers de la région, une école de gardes-malades auxiliaires fondée le 11 février 1956, fournit une maind'oeuvre locale fort appréciée.

Le 4 mars 1964, l'hôpital Saint-Sauveur est incorporé et le 15 décembre 1969, c'est le Gouvemement du Québec qui dirige les destinées de cet établissement. Actuellement, deux religieuses seulement font partie du personnel contre 25 en 1950. 


\section{LE SANATORIUM ST-JEAN DE MACAMIC $(1950)^{7}$}

La petite ville de Macamic, avec une population de 2,500 habitants est située entre Amos et LaSarre. Sa vie économique s'appuie principalement sur les emplois créés par le Centre Hospitalier St-Jean, par un bureau du Ministère des Transports et par une division de la scierie Taschereau qui fonctionne selon les demandes du marché du bois.

Le Centre Hospitalier St-Jean, qui fêtait son 30e anniversaire de fondation en 1980, est un organisme décentralisé du Ministère des Affaires sociales. C'est une institution qui aujourd'hui se consacre aux soins des maladies physiques ou des maladies mentales nécessitant des traitements de longue durée. Voyons les étapes de la transformation de ce centre de la santé depuis 1950.

Le Centre hospitalier de Macamic vit le jour sous le nom de Sanatorium de Macamic. Cette première vocation dura dix ans, soit de 1950 à 1960 : elle visait essentiellement le traitement des maladies respiratoires, particulièrement la tuberculose. Le Sanatorium pouvait hospitaliser 205 malades. Il voyait aussi au dépistage et à la prévention de la tuberculose dans la région, grâce à une roulotte munie d'un appareil radiologique qui vérifiait la présence ou l'absence du bacille infectieux. Mais d'où vient l'idée d'un Sanatorium à Macamic ? C'est suite à une requête présentée au Gouvernement de la Province de Québec par les personnes suivantes : Jacques Bouchard, courtier en assurances d'Amos, le Chanoine Zamilda Tremblay, curé de Macamic, Raoul Chamberland, gérant de la Sarre, Eugène Lambert, industriel de la Sarre et Léopold Larouche, avocat de Rouyn, demandant une charte qui les constituerait en corporation. Le Sanatorium de Macamic était constitué en corporation par lettres patentes enregistrées, le 8 mai 1947, et le bureau de direction était formé de ces mêmes personnes. Une lettre patente enregistrée le 18 octobre 1948 changeait le nom : "Corporation Sanatorium de Macamic" pour celui de la Corporation "Sanatorium St-Jean". Le 21 mai 1950, l'Assemblée des Directeurs approuve le contrat relatif à la régie interne de l'Institution confiée à la communauté des Filles de la Sagesse. Enfin, le 3 septembre 1950, avait lieu l'inauguration et la bénédiction du Sanatorium sous la présidence d'honneur du Premier ministre, Maurice Duplessis et de l'évêque d'Amos, $\mathbf{M}^{\mathrm{gr}} \mathrm{J}$.-Aldée Desmarais.

${ }^{7}$ Merci à Mme Ginette Provencher, directrice de Personnel, pour les documents qu'elle m'a fait parvenir. 
Les recherches faites pour combattre le bacille de Koch ont amené la régression de la tuberculose à travers la Province, si bien que le taux d'occupation des lits du Sanatorium St-Jean accusait une forte baisse. Le Sanatorium dut alors accepter des personnes âgées et des malades atteints de maladies chroniques. En 1975, le Gouvernement du Québec émit de nouvelles lettres patentes autorisant le Sanatorium St-Jean, connu dorénavant sous l'appellation Centre Hospitalier StJean, à utiliser 205 lits pour soins prolongés et 30 lits pour maladies respiratoires. Ce département fermera définitivement ses portes en 198182. Cette deuxième vocation ne dure que trois ans.

Dès 1978, un nouveau champ s'ouvre pour le Centre Hospitalier St-Jean : l'acceptation des apatrides handicapés mentaux. Le $3^{\mathrm{e}}$ étage est réaménagé et le personnel recyclé en vue de favoriser l'intégration des nouveaux venus. L'adaptation à cette troisième vocation fut plus difficile parce que très spécialisée. En 1977, le Centre avait ouvert un service de physiothérapie pour ses malades chroniques. Il permit ensuite à toute personne nécessitant un traitement de ce genre d'utiliser son service.

En 1979-80, dans la continuation de sa métamorphose, la direction du Centre ajoute un bassin thérapeutique de 25 pieds par 40 d'une capacité de 33,000 gallons d'eau pour les traitements par hydrothérapie dans les cas de fractures, de brûlures, d'arthrite, d'affections neurologiques ou infectueuses. Elle ouvre aussi un service d'ergothérapie pour ses patients.

Le rapport de 1981-82 indique les données suivantes : soins prolongés pour personnes âgées et handicapées physiques : taux d'occupation 90.2\%; handicapés mentaux : $98.2 \%$; pneumologie : $15.7 \%$ (département fermé en septembre 1981); expérience de trois semaines en camp d'été pour les handicapés mentaux avec des éducateurs spécialisés : expérience très concluante à poursuivre; traitements par hydrothérapie : 1,042; personnel au service des malades : 206 à temps complet et 140 à temps partiel, pour une masse salariale de $\$ 4,725,843$. En somme l'objectif du Centre vise à aménager les services le mieux possible, à améliorer la qualité des soins, à rendre agréable le séjour des malades en dépit de leur état de santé déplorable, grâce à des programmes de rééducation et de réhabilitation collectifs et individualisés. 


\section{L'HÔPITAL ST-FRANÇOIS D'ASSISE DE LA SARRE (1958).}

La Sarre existe depuis 65 ans. Cette ville aujourd'hui d'environ 8,000 âmes, s'appelait autrefois Wabakih (nom d'origine indienne) et constituait un poste important de la Compagnie de la Baie d'Hudson. Avant l'arrivée des Blancs, la tribu algonquine y règnait en maître. Des descendants des Amérindiens y habitent encore. Les Blancs plutôt francophones sont venus des Iles-de-la-Madeleine, du Lac St-Jean et de d'autres milieux québécois gagner leur vie dans les deux importantes scieries ou sur des fermes ou dans d'autres commerces florissants.

La population dynamique, entreprenante réussit à doter son milieu des services essentiels. Médecins, politiciens n'ont pas ménagé leur temps et leurs voyages pour solliciter la venue d'une jeune communauté établie à Montréal : les Oblates Franciscaines de St-Joseph. Vouée à l'enseignement et à l'hospitalisation, cette communauté, fondée en 1929, céda aux instances répétées des Docteurs Wellie Bernier et Gustave Rheault et des hommes politiques du milieu en envoyant un contingent de 17 religieuses. L'accueil chaleureux des gens, le bénévolat exercé par toutes catégories d'adultes et d'étudiants permettent aux religieuses d'ouvrir les portes de l'Hôpital St-François d'Assise le ler janvier 1958. D'une capacité de 113 lits, l'hôpital est situé à l'entrée de la ville, à environ un mille et demi du centre-ville.

À cause d'une pénurie de personnel laïque qualifié, les débuts sont difficiles. Les religieuses doivent fournir de nombreuses heures de travail supplémentaires. L'ouverture d'une école de gardes-malades auxiliaires en 1959 améliore la situation. On estime à près de 400 le nombre de jeunes filles qui ont passé par cette institution. Des spécialistes venus de Montréal et des hôpitaux de Nord-Ouest contribuent à donner satisfaction à la clientèle. Au fil des années, de nouveaux services s'ajoutent et font en sorte que La Sarre qui semblait d'abord au bout du monde (450 milles de Montréal) devient un centre agréable et plus facile d'accès.

Cet établissement, qui atteint à peine son quart de siècle d'existence, connaîtra le même sort que celui réservé aux autres hôpitaux de la Province de Québec en 1972. Quatre religieuses y travaillent encore en 1982 sous la direction d'un Conseil d'administration formé de laïques. 


\section{L'HÔPITAL DE MALARTIC (1967) ${ }^{8}$}

Située à $80 \mathrm{~km}$ de Rouyn et à $27 \mathrm{~km}$ de Val d'Or, Malartic, petite ville minière de 5,000 âmes, possède un comité de citoyens dynamique qui veille aux intérêts des occupants. Le groupe plaide si bien sa cause qu'il obtient la construction d'un hôpital répondant aux besoins de la population en obstétrique et en médecine. Les Filles de la Charité du Sacré-Coeur de Sherbrooke prennent la direction de l'hôpital de Malartic inauguré le 1er mars 1967, mais doivent quitter quelques années plus tard à cause de la pénurie de vocations.

Devant la prolifération des hôpitaux dans la région du Nord-Ouest québécois, le Ministère de la Santé et du Bien-Être juge opportun de centraliser certains services, âfin de les rendre plus efficaces. Aussi, dès 1969 l'hôpital devient le Centre Hospitalier de Malartic au sens de la loi 65 et comprend une unité de conditionnement opérant, une unité de soins courants et de soins intensifs. Une clinique externe en psychiatrie desservant la région de Malartic et un service de salle d'urgence, de laboratoire, de radiologie, se rattachent au Centre Hospitalier. L'hôpital possède une capacité de 49 lits; 138 personnes travaillent au bien-être des patients, y compris des psychiatres et des psychologues.

Au premier étage, l'unité de psychiatrie générale dispense les soins pour les bénéficiaires qui se présentent volontairement ou sur une ordonnance de cour. Le traitement a pour but d'aider les patients à obtenir un maximum d'autonomie. Au rez-de-chaussée, le conditionnement opérant dispense un traitement particulier pour les gens qui ont besoin d'acquérir des compétences au niveau de leur hygiène personnelle ou de leur travail ou des échanges en société.

En vue de favoriser la vie sociale, le Centre facilite la participation à des activités diverses : danse, sports, bingo, quilles, mini-put, séjour dans un chalet, ateliers de menuiserie et d'arts plastiques. Un aumônier catholique est en service à temps plein au Centre Hospitalier, mais les patients d'autre allégeance peuvent aussi faire appel au ministre de leur culte. L'hôpital opère un budget de trois millions et demi sous le contrôle du Ministère des Affaires sociales du Québec.

${ }^{8}$ Merci à Monsieur le curé Roméo Lapointe et à M. André Pelletier, directeur des finances du Centre Hospitalier Malartic, pour les renseignements qu'ils ont eu l'amabilité de me fournir. 


\section{FORT GEORGE (BAIE JAMES) (1930 $)^{9}$}

Transportons-nous maintenant chez la tribu des Cri de la Baie James. Quand les Pères Oblats de Marie-Immaculée Philippe Boisseau et JeanPaul Hébert jettent les bases de la mission St-Joseph à Fort George en 1922 , ils y trouvent les ministres anglicans bien installés. Fort George est une île longue de 16 milles et large de trois milles qui sépare la rivière la Grande en deux branches. Le Père Boisseau s'empresse de construire une école-hôpital, mais 11 meurt avant de voir l'édifice terminé. Le Père Meilleur continue l'oeuvre de son prédécesseur avec le Père Damase Couture. Ce dernier a foumi 45 ans de sa vie à Fort George.

L'agglomération de Fort George constitue le poste de traite le plus populeux et le plus productif de la Compagnie de la Baie d'Hudson. Malgré sept années de labeur, les Pères Oblats ne parviennent pas à amener au catholicisme un seul des six cents habitants de l'Ile. Le zèle des pasteurs anglicans et les préjugés de certains membres de la Compagnie de la Baie d'Hudson rendent les Indiens méfiants envers les missionnaires catholiques. Comme difficultés particulières à la Baie James, il faut aussi mentionner l'adaptation à la langue et à la mentalité des indigènes et leur nomadisme dû à leurs moyens de subsistance : la chasse et la pêche.

En 1929, $\mathbf{M}^{\mathrm{gr}}$ Louis Rhéaume, évêque d'Haileybury et le Père Saindon, provincial des missions de la Baie James, pressent le Conseil général des Soeurs Grises de la Croix d'envoyer à Fort George quatre religieuses seconder le travail des Pères Oblats. La demande acceptée, les fondatrices arrivent à Moosonee, terminus du chemin de fer Ontario Northland, le 10 mai 1930. Elles prennent ensuite un bateau qui leur fait parcourir les 300 milles séparant Moosonee de Fort-George.

Les premiers services se font sous la tente. Peu après leur arrivée, le 10 juillet 1930, une épidémie de grippe sévit dans le village. Les Soeurs se donnent sans compter pour soigner les nombreux malades. Le 15 août $1933 \mathrm{M}^{\mathrm{gr}}$ Rhéaume fait sa visite pastorale à Fort George. Il constate une seule conversion et apprend que le bon Dr Wall réussit

\footnotetext{
9 Sources consultées : Archives de la Maison-Mère des Soeurs de la Charité d'Ottawa; Chroniques et procès-verbaux de la communauté de l'Hôpital de Fort George; Bulletin communautaire des Soeurs de la Charité d'Ottawa, Vol. 7, no 3; Sr PaulÉmile, s.c.p., Amiskwaki, (Terre du Castor), Les Soeurs Grises à la Baie James, 1952, p. 234-271; Sr Paul-Émile, s.g.o.
} 
à faire subventionner l'hôpital de la mission par le Gouvernement, en dépit des difficultés qu'occasionne le fanatisme ambiant. La mission catholique ne possède aucun revenu. Le Conseil épiscopal d'Haileybury et le Conseil provincial des Oblats hésitent à maintenir ouverte l'oeuvre de Fort George. Confiant dans la Providence et dans la générosité des missionnaires, Monseigneur revient le 18 du même mois avec le Père Henri Belleau et décide de laisser les missionnaires continuer leur travail malgré leur extrême pauvreté.

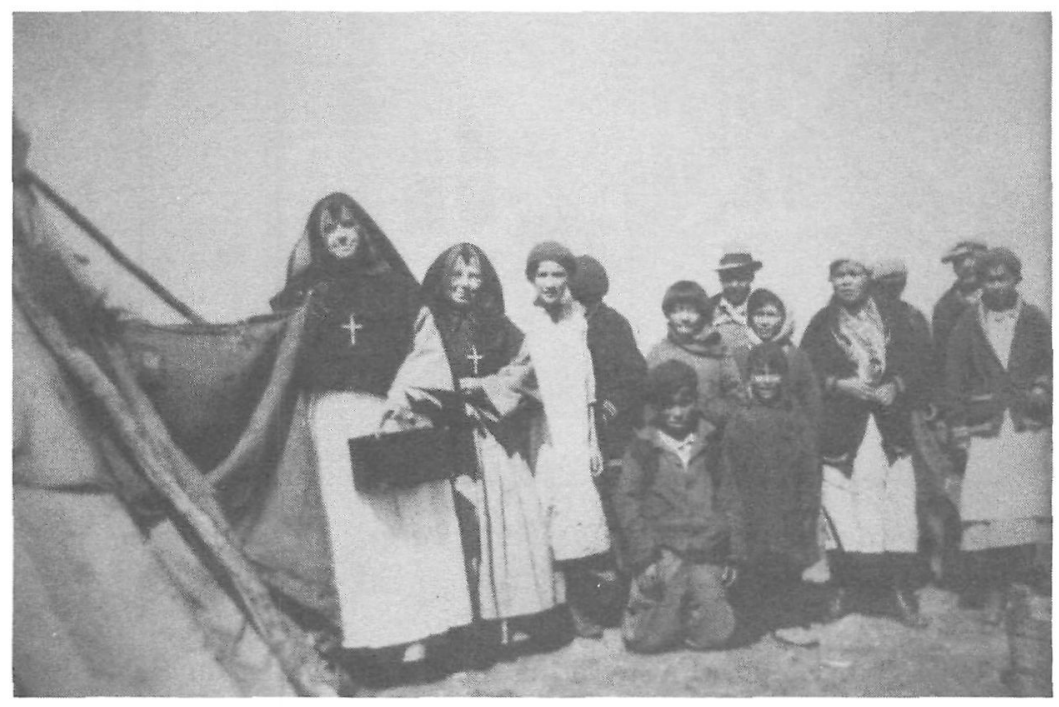

Visite des malades sous la tente sur l'Ile de Fort George (ca 1934-35).

En 1934-35, les chroniques de l'hôpital fournissent les statistiques suivantes : 43 patients admis à l'hôpital (il leur faut de l'audace, puisqu'ils sont menacés de se faire couper les vivres par les pasteurs anglicans); 129 malades soignés sous la tente; 1200 consultations et traitements au dispensaire. La bonté, la patience, le zèle, la prière des religieuses font tomber les craintes des Indiens. Vu le travail extraordinaire fourni par les soeurs infirmières, le Dr Wall obtient, en 1935, un octroi du Gouvernement fédéral pour l'hôpital de Fort George. De son côté, le chef indien Tchiskamash visite l'école et l'hôpital catholiques et se montre très satisfait.

On peut se demander pourquoi le Vicariat apostolique de la Baie James, pris en charge en 1940 par $\mathrm{M}^{\mathrm{gr}}$ Belleau, assume-t-il le lourd fardeau des frais de construction et d'administration d'un hôpital catholique, quand le Gouvernement fédéral construit à ses frais pour les In- 
diens? C'est la tradition catholique de soulager les misères corporelles et spirituelles. L'Église veut respecter les Indiens qui acceptent de recevoir les sacrements et d'être aidés par la prière commune.

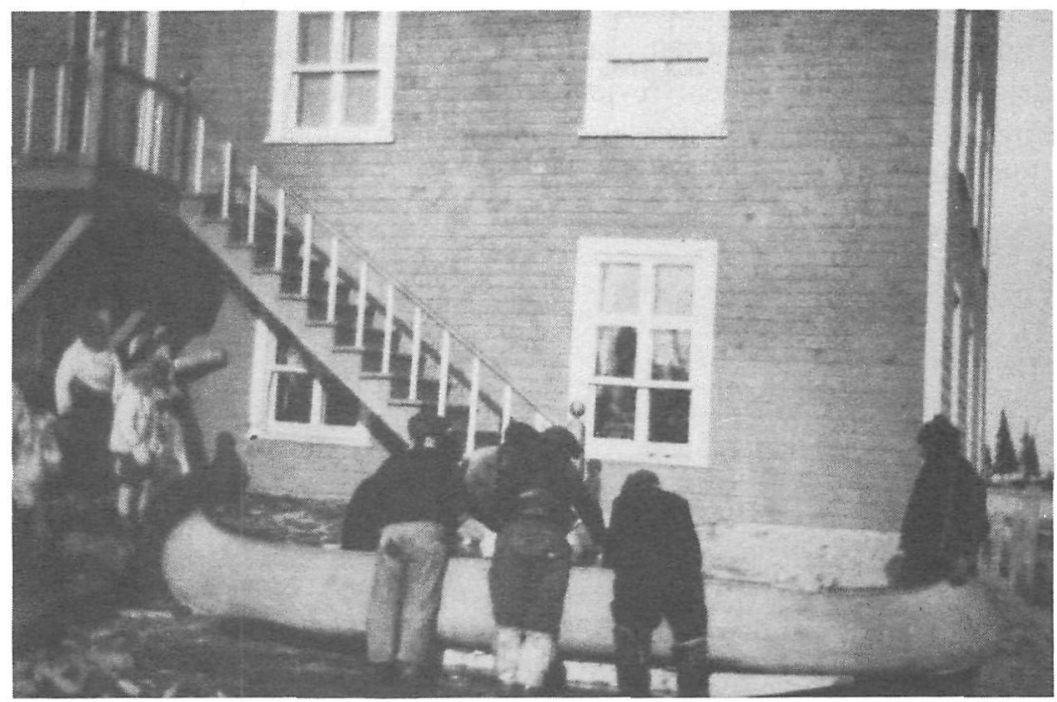

«Ambulance» de l'ancien hôpital de Fort George : un canot monté sur un traîneau (ca 1934-35).

À cette époque, un Ministre de la Santé nationale et du Bien-être assure le Vicaire apostolique qu'il respecte la ligne de conduite établie par les missionnaires, à savoir que les Indiens ont le libre choix de l'hôpital où ils veulent être soignés. En 1946, le gouvernement fédéral construit un hôpital d'État pour les Indiens, parce que le Ministère des Affaires indiennes accorde des subsides aux familles autochtones.

Dans les chroniques de l'hôpital catholique, on note des épidémies de rougeole, d'influenza, de pneumonie en 1942 et en 1949, pendant lesquelles toutes les Soeurs de la mission se dépensent sans compter sous la direction de Soeur Ste-Rolande en 1942 et de Soeur Marie-Elmire en 1949. On appelait cette dernière l'Ange Blanc du Nord.

Voici les statistiques de 1942 à 1951 pour l'hôpital catholique : 1849 Indiens hospitalisés, 548 Blancs hospitalisés, 60 naissances, 5,274 visites sous la tente, 51,285 visites au dispensaire, 1,895 obturations ou extractions de dents, 2,048 radiographies. Vingt ans sont passés et un seul néophyte a accepté de faire partie de l'Église catholique. Mystère de l'attente!... Le travail des religieuses n'est pas rémunéré. Les Pères 
Oblats leur fournissent logement, nourriture, vêtements et autres dépenses inhérentes à leur tâche professionnelle.

Après vingt ans, la mission a réussi à sortir de la misère du début. Le 9 juillet 1950, grâce à une modeste subvention des gouvernements fédéral et provincial obtenue par l'intermédiaire du Vicaire apostolique, $\mathbf{M}^{\mathrm{gr}}$ Belleau, la mission ajoute un nouvel édifice d'un étage mesurant $160 \times 40$ pieds. Ce nouvel hôpital attenant au Pensionnat-école contient 32 lits, un département de radiologie, une salle d'opération, un laboratoire, une pouponnière, un système de chauffage central, l'eau chaude et l'eau froide, un service d'intercommunication, une aile réservée aux tuberculeux. Ce nouvel hôpital est sans conteste, la perle de la région.

Lors de la visite de Fort George par Mère St-Paul, supérieur générale des Soeurs de la Charité d'Ottawa en 1958, celle-ci constate que les sept à huit cents Indiens sont fermés à la présence de l'Église catholique. La visiteuse se rend compte cependant que l'Indien de Fort George apprécie l'enseignement et les soins médicaux donnés par les religieuses.

Les registres 1957-58 révèlent les données suivantes : 182 malades admis à l'hôpital, 1080 au dispensaire, 1600 soignés sous la tente. Neuf religieuses oeuvrent à la mission de Fort George. Les entrées à l'hôpital continuent de s'accroître, si bien qu'en 1962, les soeurs ne peuvent plus suffire à la tâche. La mission engage des infirmières laïques. Des jeunes Indiennes mettent aussi leurs talents au service des malades. En 1964, bonne nouvelle! Le diocèse de Moosonee engage un médecin-chirurgien résident : le Dr Louis Lavallée, pour Fort George. Dans les débuts, les religieuses infirmières remplissaient toutes les urgences : chirurgie, extractions de dents, médecine générale. Le Dr James Sands suivra en 1967 et le Dr Charles Dumont en 1969. À cette date, la population atteint 1200 âmes.

L'espace manquant, le Ministère des Richesses naturelles du Québec construit un hôpital moderne d'une capacité de 30 lits sur le côté ouest entre le village des Indiens et la mission : c'est l'hôpital Chisasipish (la rivière Grande). La loi de 1969 du Gouvernement du Québec affecte aussi l'hôpital de la mission. Le 14 septembre 1970, un laïque, M. de Bellefeuille, devient le directeur, assisté de M. Bernard Piché, comptable. Après 1971, aucune religieuse ne fait partie du personnel, faute d'effectifs disponibles. 
Depuis lors, les travaux gigantesques des chantiers LG2, LG3 et LG4 font disparaître l'Ile de Fort George sous les eaux de la rivière La Grande. Maisons, institutions ont été démolies et reconstruites dans un endroit plus sécuritaire. Deux Pères Oblats ont suivi les Indiens dans leur exode. C'est une autre histoire qui commence.

\section{CONCLUSION}

Ce survol des institutions hospitalières du Nord-Ouest québécois révèle une constante : des communautés religieuses répondent aux besoins du milieu comme service d'Église accepté dans la foi et la confiance. C'est toujours un défi à relever. Si l'on se reporte à l'histoire de l'Église catholique au Canada, quelle floraison de femmes vouées à une portion choyée par le Seigneur : les démunis de la santé! Elles ont gardé actif le charisme de compassion possédé par leurs fondatrices. Si elles ont perdu la gestion temporelle des hôpitaux, un certain nombre de religieuses travaillent encore dans l'ombre et maintiennent vivace l'esprit qui assure une continuité dans le temps et dans l'espace.

Hommages respectueux à celles qui ont porté le flambeau, qui l'ont transmis d'âge en âge, et qui continuent silencieusement, mais avec non moins d'audace, une oeuvre qui fait la gloire de l'Église catholique de notre pays!

Soeur Simone GareaU, s.g.c. St-Charles de Mandeville*

*NDLR : Soeur Gareau a été principale de l'École normale de VilleMarie de 1959 à 1964 et membre du Conseil d'administration de l'Hôpital de Noranda de 1965 à 1972; elle est aujourd'hui agent de pastorale à St-Charles-de-Mandeville (Cté Berthier). 